\title{
LÉGISLATION
}

\section{Le courant électrique importé de Suisse et la taxe d'importation Arrêt de la Cour de Cassation du 5 Juillet 1927}

\author{
par Paul Bougault, Avocat à la Cour d'Appel de Lyon
}

\begin{abstract}
L'auteur fait connaître, dans l'étude que l'on va lire, non seulement le texte de l'arrêt qui a déclaré le courant électrique "marchandise taxable à l'importation", mais encore les moyens que le contribuable a fait valoir pour échapper à cette sujétion nouvelle, et qui ont été, malheureusement, rejetés par la Cour de Cassation.
\end{abstract}

Faits ayant donné lieu au procès et textes à invoquer. - La Société des Houillères de Ronchamp fait venir de Suisse du courant électrique qui franchit la frontière et vient accomplir en France son effet utile dans plusieurs distributions. Ce courant est-il soumis à la taxe dite d'importation qui est édictée par l'article 72 de la loi du 25 juin 1920, dont le premier alinéa est ainsi conçu : "Les importations d'objets ou de marchandises " sont soumises quel que soit l'importateur, à un droit de $1 \%$ qui " sera liquidé sur la valeur desdits objets ou marchandises, " droits de douane et de consommation ou de circulation com" pris ; .....l'impôt sera perçu, les contraventions seront punies, "les poursuites seront effectuées et les instances instruites et " jugées, comme en matière de douane et par les Tribunaux " compétents en cette matière. "

La Société avait soutenu devant le Tribunal de Lure, différents moyens que nous n'avons point à analyser, puisque nous les retrouverons ci-dessous dans la discussion de l'arrêt de la Cour Suprême du 5 juillet 1927, qui a rendu définitif le jugement du Tribunal Civil de Lure du 10 avril 1924.

Disons seulement que ces moyens paraissaient bien fondés, ils ont été repris devant la Cour Suprême, et se divisent en deux séries :

Dans la première, on peut classer plusieurs moyens ayant trait à la discussion de différents textes de la loi du 25 juin 192 , d'après lesquels le courant électrique ne saurait être ni taxé, ni taxable : dans la seconde, une seule question dite de prescription a été envisagée.

Le premier moyen consistail ì dire : l'article 72 de la loi du 25 juin 1920 vise les objets; or, le courant électrique n'est pas un " objet ». On ne touche pas, on ne voit pas, on ne pèse pas un kilowatt ; de plus, à supposer que ce soit un objet, il faut reconnaître qu'on ne le trouve inscrit sur aucune liste douanière. Or, la taxe d'importation est, en quelque sorte, une taxe succédance de la taxe de douane.

La Cour Suprême, inexorablement, a répondu par la lecture du tarif et n'a attaché aucune importance à la "définition " qui avait élé tentée par la Société, au sujet de " la nature tangible de l'objet ». Elle a constaté simplement qu'un fil faisait entrer en France, une chose utilisée en France, et cela lui suffit pour dire que, bien que ce soit un courant électrique, la large extension dont le mot "objet " est susceptible, permet sans difficulté de l'appliquer au courant.
Le second moyen, beaucoup plus faible, était basé sur une prétendue insuffisance de la décision attaquée : en soumettant le courant à la taxe qui frappe une marchandise, le Tribunal n'aurait pas constaté que cette marchandise n'avait pas été fabriquée en France.

Du moment que la Cour de Cassation, en réponse au premier moyen, avait constaté que le mot " objet " suffisait pour légitimer la taxation, il n'y avait rien à espérer du second moyen.

Le troisième moyen était beaucoup plus sérieux : à maintes reprises, des auteurs ont admis et expliqué que, dans une certaine mesure, on peut assimiler la taxe d'importation à la taxe sur le chiffre d'affaires; la première n'a été créée que pour empêcher les commerçants d'éluder la secc.ade, en achetant des marchandises qui, fabriquées à l'étranger, n'auraient pas eu un prix de revient grevé de la taxe sur le chiffre d'affaires. Or, on sait que les concessionnaires d'un service public sont exemptés de la taxe par l'article 60 de la loi du 25 juin 1920. La pénétration du courant doit profiter (au titre de l'importation), de l'exonération dont jouit la vente de ce courant quand elle est opérée par un service public. La Cour de Cassation a répondu qu'elle ne voulait, en aucun cas, confondre la taxe sur le chiffre d'affaires et la taxe d'importation; car celle-ci, tout en ayant un tarif identique, n'est point vis-à-vis de l'autre, en étal de dépendance. Chacune a même la sphère qui lui est propre : l'article 72 serait le texte organique de la taxe d'importation, comme l'article 59 serait celui du chiffre d'affaires, et la Cour remarque que cet article 59 est absolument muet sur tout ce qui est relatif à la pénétration d'un objet en France, puisqu'il ne vise. que des transactions purement commerciales.

La seconde série ne contient qu'un moyen essentiellement différent de tous les autres : il était destiné à limiter le risque de la Société au moyen de la prescription : "A supposer que je sois " taxable, disait la Société, je ne pourrais être recherchée que " jusqu'à concurrence du courant ayant pénétré en France dans "le cours de la dernière année. En effet, l'article 25 du titre XIII " du décret du 6-22 août 1791 déclare ce qui suit : la régie sera " non recevable à former aucune demande en paiement des " droits, un an après que lesdits droits auront dû être payés. "

La Cour de Cassation a rejeté ce moyen de prescription, en déclarant que la Société ne lui paraissail pas avoir été de bonne foi dans cette affaire.

En consécquence, elle a confirmé le jugement du 10 avril 1924 
dans les termes que l'on va lire. Elle a même rendu le même jour un autre arrèt dans le mème sens et sur le mème sujet à l'encontre d'une autre Société : celle des Forces Motrices de la Goulé de Saint-Insiér.

Texte de l'arrêt. - La Cour, sur le premier moyen :

Attendu qu’à bon droit le jugement attaqué a condamné la Société des Houillères de Ronchamp à payer à l'Administration des Douanes l'impôt établi par l'article 72 , de la loi du 25 juin 1920, à raison de la valeur de l’énergie électrique provenant de Suisse et importée en France par ladite Société, notamment à fin de distribution et vente à ses abonnés;

Attendu, en effet, qu'aux termes de l'art. 72 est assujettie à la taxe, toute importation d'objets ou de marchandises; que des expressions, dont la portée est générale 't extensive,s'appliquent à l'énergie électrique, produit industriel, constiluant un objet ou marchandise, au sens matériel du terme, et susceptible d'être taxé fiscalement;

Attendu que le pourvoi prétend, à la vérité, que le droit établi par l'art. 72 étant assimilable au droit de douane, ne peut porter sur un objet qui ne figure pas au tarif ;

Attendu que l'art. 72 et les textes qui en règlent l'application visent les objets et marchandises quels qu'ils soient et d'où qu'ils viennent;

Attendu, en effet, que l'arrêté du 28 août 1920, relatif notamment à la perception des taxes instituées par les art. 63 et 72 de la loi du 25 juin 1920 dispose dans son art. $1^{\text {er }}$ que ces taxes sont applicables non seulement à toutes les importations de l'étranger, mais aussi à celles de l'Algérie, des colonies et des possessions françaises, bien que les marchandises provenant de l'Algérie et des colonies assimilées ne soient pas en principe passibles des droits de douane ; qu'ainsi le moyen n'est pas fondé ;

Sur le deuxième moyen et d'abord sur la fin de non recevoir qui lui est opposée et tirée de sa nouveauté ;

Attendu que ce moyen est en corrélation étroite et direct avec les motifs du jugement attaqué, adoptant lui-même ceux du premier juge, que, par suite, il est recevable ;

Rejette la fin de non recevoir ;

Attendu, d'après le pourvoi, que le jugement attaqué ne constatant pas que le courant introduit par la Société en France, avait été acheté en Suisse alors que la taxe percue à l'importation suppose nécessairement qu'il y a eu un achat à l'extérieur, manquerait de base légale;

Mais attendu que le fait visé, qui donne lieu à la taxe, est l'importation d'un objet quelconque, sans qu'il y ait à s'occu- per d'aucune autre condition ; que la matière étant de droit strict il faut s'attacher au texte même de la loi qui est précis el ne distingue pas ; que le moyen n'est donc pas fondé ;

Sur le troisième moyen :

Attendu que le troisième moyen ne l'est pas davantage;

Attendu, en effet, que, d'après le pourvoi la laxe de l'art. 72 sur l'importation et celle de l'art. 59 sur le chiffre d'affaires n'étant qu'un même impôt, les concessionnaires de services publics, exempts (art. 60) du paiement de la taxe sur le chiffre d'affaires doivent l'être également de la la..e sur l'importation, du moins pour les quantités d'électricité employées par les services publics concédés ;

Mais, attendu que la taxe à l'article 72, quoique d'un taux égal au taux de la taxe sur le chiffre d'affaires, n'est pas sous la dépendance de celte dernière taxe; que ces deux taxes, recouvrées par des administrations différentes, relevant de juridictions différentes, diffèrent également quant à leur nature ; que l'art. 72 frappe les objets à raison du fait matériel de leur introduction en France, d'après leur valeur propre, intrinsèque, el abstraction faite de leur utilisation; que l'impôt établi par l'art. 59 frappe au contraire, non pas les objets ou marchandises importées, mais l'ensemble des transactions commerciales, c'est-à-dire le chiffre d'affaires;

Attendu que l'article 60 , invoqué par le pourvoi comme portant exonération de la taxe, vise expressément celle sur le chiffre d'affaires et ne contient aucune disposition relative à la taxe frappant les objets ou marchandises importés ;

Sur le quatrième moyen :

Attendu que si l'article 25, titre XIII, de la loi des 6-22 août 1791 dispose que l'Administration des douanes n'est recevable à former aucune demande en paiement de droits un an après que ces dits droits auraient dû être payés, c'est à la condition qu'il s'agisse d'un importateur de bonne foi ;

Attendu que le jugement attaqué constate que la Société des Houillères de Ronchamp qui n'a pas fait de déclaration à la douane pour l'énergie électrique provenant de Suisse et introduite en France depuis le $1^{\text {er }}$ juillet 1920, n'était pas de bonne foi; que cette appréciation échappe au contrôle de la cour de Cassation; que c'est donc à bon droit que le jugement attaqué a déclaré que le droit commun était applicable, et que l'action de la Douane pouvait s'exercer pendant trente ans, à compter de la constatation des faits;

Par ces motifs : rejette...

MM. Seligman, prés.; Lénard, rapp.; Paul Matter, av. gén. ; Mes Chassagnade-Belmin et Dambeza, av. 